\title{
Bifosfonati e disfunzione renale
}

Giornale di Tecniche Nefrologiche e Dialitiche 2018, Vol. 30(3-4) 194-203

(C) The Author(s) 2019

Article reuse guidelines:

sagepub.com/journals-permissions

DOI: $10.1177 / 0394936218823508$

journals.sagepub.com/home/gtn

\author{
Giulio Distefano', Luca Zanoli², Luca Di Lullo3, \\ Antonio Basile' e Antonio Granata ${ }^{4}$
}

(3)AGE

\begin{abstract}
Disphosphonates and renal impairment

Osteoporosis and chronic kidney disease (CKD) are two frequent pathological conditions in the adult and geriatric population and often coexist. These conditions have a significant negative impact on the quality of bone tissue, complicated by the coexistence of CKD and metabolic bone disease (CKD-MBD) and a greater incidence of fractures than the general population. Bisphosphonates are a class of drugs effective in preventing bone fractures by inhibiting tissue resorption by osteoclasts. Efficacy and tolerability have also been extensively studied in patients with mild CKD, but doubts remain on the renal safety profile in patients with advanced and terminal chronic nephropathy. In this article we conducted a review of the evidence currently available on PubMed. The studies published up to now on bisphosphonates approved for oral or parenteral use demonstrate that there are no significant effects on long-term renal function when administered to patients with eGFR values $>30 \mathrm{~mL} / \mathrm{min} / 1.73 \mathrm{~m}^{2}$ or $>35 \mathrm{~mL} / \mathrm{min} / 1.73 \mathrm{~m}^{2}$. Nevertheless, acute, but for the most transient, changes of renal function are possible after endovenous infusion of ibandronate and zoledronic acid. Since controlled trials in patients with eGFR $>30 \mathrm{~mL} / \mathrm{min} / 1.73 \mathrm{~m}^{2}$ are not available, it is not possible to comment on the safety profile in this population.
\end{abstract}

\section{Keywords}

Diphosphonates, Osteoporosis, Chronic Kidney Disease-Mineral and Bone Disorder, Acute kidney injury, Bone remodeling

\section{Introduzione}

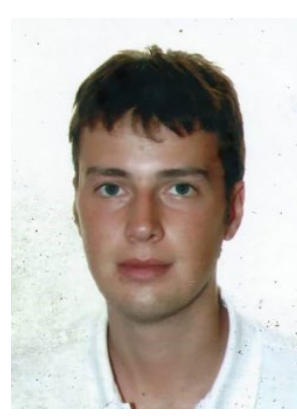

L'osteoporosi e la malattia renale cronica (chronic kidney disease, CKD) sono due condizioni patologiche spesso coesistenti, e rappresentano un problema di sanità pubblica per via dell'elevata prevalenza nella popolazione geriatrica. È noto che il rischio di fratture da fragilità è notevolmente incrementato nel paziente nefropatico affetto da osteoporosi, con conseguente significativo aumento, in questa popolazione, del rischio di ospedalizzazione e con conseguente aumento della disabilità fisica, della mortalità e dei costi sociali e sanitari. ${ }^{1}$ I bifosfonati rappresentano una terapia di prima linea nella popolazione generale, ma nel paziente con insufficienza renale cronica la prescrizione di questi farmaci richiede più prudenza ed è gravata da considerazioni di tipo farmacocinetico e da un quadro di sicurezza ancora poco chiaro.

\section{Scopo}

Scopo di questo lavoro è fornire una revisione critica delle acquisizioni presenti nella letteratura internazionale

\footnotetext{
'Dipartimento di Scienze Mediche e Chirurgiche e Tecnologie Avanzate, Università di Catania, Italia

2Dipartimento di Medicina Clinica e Sperimentale, Università di Catania, Italia

3U.O.C. Nefrologia e Dialisi - Ospedale "L. Parodi - Delfino",

Collereffo (Roma), Italia

${ }^{4}$ U.O.C. Nefrologia e Dialisi - P.O. “San Giovanni di Dio”, Agrigento, Italia

Corresponding author:

Dott. Giulio Distefano, Dipartimento di Scienze Mediche e Chirurgiche e Tecnologie Avanzate, Università di Catania, Via B. Croce n 6, 97100

Ragusa (RG), Italy.

E-mail: giuliodistefano@gmail.com
} 
relativamente alle caratteristiche farmacologiche dei bifosfonati e al loro impiego nella popolazione affetta da nefropatia cronica ed acuta.

\section{Metodo}

È stata eseguita una ricerca attraverso il database PubMed nei campi dell'osteoporosi, della malattia renale cronica, della malattia metabolica dell'osso, della qualità dell'osso, del rischio di fratture, della farmacologia dei bifosfonati e del profilo di sicurezza dell'uso dei bifosfonati in varie popolazioni. Sono state tratte informazioni rilevanti da articoli originali, dalle linee guida di riferimento e da pre-cedenti revisioni; ulteriori lavori sono stati valutati dall'analisi della bibliografia degli articoli trovati. Sono state infine selezionate pubblicazioni rilevanti per lo scopo proposto per questa review.

\section{Definizione e dimensione della problematica}

L'osteoporosi e la CKD sono due condizioni patologiche ampiamente rappresentate nella popolazione generale, con particolare riferimento alla popolazione adulta e anziana, e sono due dei maggiori problemi di sanità pubblica. In letteratura è stato riportato che la prevalenza di End-Stage Renal Disease (ESRD) è superiore a 1,000 casi su un milione di abitanti in Italia con un'incidenza di circa 150 nuovi casi su milione di abitanti, mentre negli Stati Uniti la prevalenza è di oltre 1,800 casi su un milione di abitanti. Più incerti sono $\mathrm{i}$ dati relativi agli stadi precoci ed asintomatici della $\mathrm{CKD}$, con una prevalenza stimata tra $1^{\prime} 8 \%$ ed il $16 \%$ sulla popolazione mondiale. ${ }^{2}$ L'osteoporosi è definita da una densità minerale ossea (BMD) (espressa in $\mathrm{g} / \mathrm{cm}^{2}$ ), valutata con la tecnica Dual Energy X-Ray Absorptiometry (DEXA), inferiore a 2.5 deviazioni standard rispetto al giovane adulto dello stesso sesso e della stessa etnia. ${ }^{3}$ L'osteoporosi è una malattia sistemica del tessuto scheletrico caratterizzata da riduzione della massa ossea e dalla compromissione dell'architettura istologica scheletrica avente come conseguenza un aumento del rischio di frattura da fragilità. ${ }^{4}$ Nell'osteoporosi si determinata uno squilibrio tra i processi di neoformazione e riassorbimento osseo, con conseguente assottigliamento dell'osso corticale e delle trabecole nell'osso spugnoso e quindi perdita della massa ossea. La BMD rappresenta una componente fondamentale nella definizione della resistenza ossea. Altro elemento che concorre a definire la resistenza ossea è la qualità dell'osso (bone quality, $\mathrm{BQ}$ ), rappresentata dalla risultanza di diverse componenti tra cui le caratteristiche istologiche, il turnover osseo e la mineralizzazione: 5 tali caratteristiche possono essere alterate in corso di nefropatia cronica. In effetti, la definizione di osteoporosi oggi attualmente accettata è certamente valida per la popolazione generale, ma non è chiaro se sia altrettanto applicabile alla popolazione nefropatica. In questa popolazione, infatti, possono essere presenti quadri di severe alterazioni morfofunzionali pur in assenza di alte-razioni significative della BMD: le alterazioni della $\mathrm{BQ}$ sono correlate con un incremento del rischio di fratture da fragilità. Tale considerazione è alla base del concetto di "osteoporosi uremica", recentemente proposto in letteratura per esprimere l'insieme delle alterazioni della struttura e della funzione ossea in un paziente uremico, ${ }^{1}$ sebbene ancora non siano stati definiti criteri diagnostici utilizzabili nella pratica clinica.

Sia l'osteoporosi che la CKD sono due condizioni la cui prevalenza è maggiore nella popolazione adulta e anziana, ed è intuitivo che le due condizioni possono coesistere nello stesso paziente. È stato descritto che le fratture da fragilità nel nefropatico sono fino a 14 volte più frequenti, interessano pazienti mediamente più giovani e sono associate a maggior mortalità e tempi di ospedalizzazione più lunghi rispetto ai pazienti non nefropatici. ${ }^{1} \mathrm{Nel}$ paziente nefropatico cronico le alterazioni metaboliche e strutturali a carico dell'osso sono più complesse che nella popolazione generale per via della coesistenza di malattia metabolica dell'osso associata alla CKD (CKD-MBD); i recenti aggiornamenti delle linee guida KDIGO sulla gestione della CKD-MBD suggeriscono la valutazione della BMD nel paziente con eGFR inferiore a $60 \mathrm{~mL} / \mathrm{min} / 1.73 \mathrm{~m}^{2}$ per la corretta stratificazione del rischio. ${ }^{6}$ Sia la CKD-MDB ad alto turnover che quella a basso turnover hanno un impatto significativo sulle caratteristiche meccaniche dell'osso. Nelle condizioni in cui predomina il riassorbimento osseo, si osserva una perdita di massa ossea con associata riduzione della resistenza dell'osso. ${ }^{7}$ Queste modifiche determinano una maggior incidenza di fratture nei pazienti nefropatici attraverso molteplici meccanismi patogenetici solo in parte noti, compresa l'azione di tossine uremiche (indosisil solfato, p-cresilsofato, AGE) che determinano incremento dello stress ossidativo alterando in maniera significativa la produzione di collagene ed avendo come risultato finale un peggioramento delle caratteristiche della qualità dell'osso. ${ }^{1}$

\section{Caratteristiche farmacologiche e ruolo dei bifosfonati nel trattamento dell'osteoporosi}

I bifosfonati sono farmaci che trovano indicazione nel trattamento dell'osteoporosi, dell'ipercalcemia e delle lesioni metastatiche dell'osso; in relazione al loro meccanismo di azione sono considerati "farmaci anti-riassorbitivi" (come il denosumab, i SERM e gli estrogeni) poiché interferiscono con i processi di riassorbimento osseo, differenziandosi concettualmente dai "farmaci osteo-anabolici" (paratormone e l'analogo teriparatide) che incrementano la sintesi di tessuto osseo. I bifosfonati si caratterizzano per essere analoghi chimici stabili del pirofosfato in cui il gruppo P-O-P è sostituito 
Tabella I. Classificazione dei principi attivi appartenenti alla classe dei bifosfonati.

\begin{tabular}{ll}
\hline Non-aminobifosfonati & $\begin{array}{l}\text { Clodronato, Etidronato } \\
\text { Aminobifosfonati }\end{array}$ \\
Aminobifosfonati eterociclici & $\begin{array}{l}\text { Ibadronato } \\
\text { Risedronato, Zoledronato }\end{array}$ \\
\hline
\end{tabular}

dal gruppo P-C-P. Sono state distinte due classi di bifosfonati (tabella 1): i "nitrogen containing bisphosphonates", o aminobifosfonati, e i "non-nitrogen containing bisphosphonates" o non-aminobifosfonati. Della prima classe sono in commercio alendronato, ibandronato e pamidronato, mentre della seconda classe sono in commercio clodronato ed etidronato; il risedronato e l'acido zoledronico, appartenenti anch'essi alla classe degli aminobifosfonati, sono anche definiti bifosfonati eterociclici. Il meccanismo d'azione dei bifosfonati è stato solo in parte chiarito in quanto sembra che le diverse tipologie abbiano diversa farmacodinamica. In particolare, è stato descritto che negli osteoclasti e negli osteociti gli aminobifosfonati inibiscono la via dell'acido mevalonico mentre i non-aminobifosfonati inibiscono le vie intracellulari dipendenti dall'adenosina trifosfato fino all'induzione dell'apoptosi. La biodisponibilità dei bifosfonati è relativamente scarsa dopo somministrazione per via orale, seppur variabile in relazione al principio attivo utilizzato. I bifosfonati non subiscono metabolismo di prima fase e pertanto giungono immodificati al sito di azione, e l'assorbimento da parte degli osteoclasti è differente in relazione al principio attivo usato e correlato con i markers di turnover osseo. ${ }^{8}$ Oltre all'attività citotossica sugli osteoclasti, è stata descritta in vitro per alcuni bifosfonati l'inibizione dell'apoptosi di osteociti ed osteoblasti e la promozione di cellule progenitrici midollari degli osteoblasti: il significato biologico e l'eventuale impatto clinico di tali riscontri non è ancora stato chiarito. ${ }^{9}$ L'escrezione è esclusivamente renale, sia per filtrazione glomerulare che per trasporto attivo, in particolare a livello del tubulo contorto prossimale. L'escrezione epatica è considerata invece pressoché nulla, come dimostrato da ormai datati studi in vivo su cavia dopo somministrazione endovenosa (ev) di alendronato radiomarcato. ${ }^{10,11} \mathrm{Il}$ meccanismo di escrezione renale è controverso. A livello tubulare l'escrezione di alendronato non è inibita né dalla somministrazione di inibitori selettivi dei trasportatori degli anioni né dei trasportatori dei cationi, come evidenziato dalla mancata risposta sia alla somministrazione di acido paraamminoippurico o probenecid che di cimetidina, mentre la sua escrezione è inibita dalla contemporanea sommini-strazione di un altro bifosfonato. ${ }^{12}$ Per i bifosfonati somministrati oralmente (peros) l'esofagite e la gastrite su base irritativa rappresentano effetti avversi relativamente comuni, mentre le somministrazioni ev sono spesso associate a febbre, leucopenia e dolori ossei diffusi. L'osteonecrosi del mascellare, la fibrillazione atriale, la tossicità oculare e la nefrotossicità rappresentano effetti avversi rari ma temibili. ${ }^{8}$ Il clodronato è prescrivibile in Italia per pazienti con mieloma multiplo, osteolisi tumorale, iperparatiroidismo primitivo e trattamento o prevenzione dell'osteoporosi post-menopausa, è stato il primo farmaco di questa classe ad essere disponibile sia per uso orale che parenterale. Rilevanti effetti sulla massa ossea sono stati descritti già a sei mesi dall'inizio della somministrazione settimanale, mentre il follow-up a tre anni ha dimostrato una riduzione dell'incidenza di fratture vertebrali del $46 \%$ rispetto ai controlli; al clodronato è inoltre riconosciuto un effetto antalgico. ${ }^{13}$ L'alendronato è un farmaco rivolto a pazienti sia in prevenzione primaria che secondaria in grado di ottenere una riduzione del rischio relativo di nuove fratture del $50 \%$. Il farmaco si è dimostrato efficace in un ampio spettro di condizioni cliniche: prevenzione primaria, prevenzione secondaria, fratture vertebrali, fratture non vertebrali ed osteoporosi da corticosteroidi. Pur con end-point secondari, l'uso del farmaco è stato testato anche per periodi di trattamento pro-lungati. Compatibilmente con la tollerabilità del farmaco (che è complessivamente buona soprattutto se assunto con le modalità corrette) e l'aderenza del paziente alla terapia, l'obiettivo di un trattamento protratto nel tempo è da perseguire. Anche l'utilizzo del risedronato ha evidenziato riduzione delle fratture vertebrali e non vertebrali con dati quantitativamente sovrapponibili a quelli dell'alendronato. Per l'ibadronato è documentata una chiara efficacia sulla riduzione delle fratture vertebrali, ma non è direttamente dimostrata l'efficacia sulle fratture non-vertebrali. Nonostante questa importante limitazione, il farmaco è utile nelle forme di osteoporosi a prevalente localizzazione vertebrale anche in singola somministrazione mensile. ${ }^{14} \mathrm{Il}$ profilo di sicurezza del pamidronato somministrato per via ev è stato descritto in uno studio di Palomo e colleghi nel $2015 .{ }^{15}$ Gli autori hanno proposto una modifica al protocollo standard per l'osteogenesi imperfecta in una popolazione pediatrica. La somministrazione di pamidronato ev ha seguito il seguente schema: $2 \mathrm{mg} / \mathrm{kg}$ in $2 \mathrm{~h}$ ogni 4 mesi per un anno. ${ }^{15}$ Già noto per il trattamento delle metastasi ossee e del mieloma, l'acido zoledronico è stato studiato per la terapia dell'osteoporosi, alla dose di $5 \mathrm{mg}$ ev una volta all'anno. Le credenziali di efficacia del farmaco sono molto buone, anche se è stata segnalata tossicità cardiaca (episodi di fibrillazione atriale) e renale (insufficienza renale acuta reversibile). Pur non essendo stati dimostrati nel trial episodi di osteonecrosi della mandibola, l'acido zoledronico rimane pur sempre il farmaco principalmente responsabile di questo effetto collaterale. La particolare modalità di somministrazione (ev annuale) impone nuove considerazioni sulla nicchia di appropriatezza dell'uso di questo farmaco, che non è ancora definita, e sul setting assistenziale (necessariamente ospedaliero). 


\section{Bifosfonati e nefrotossicità}

La prescrizione dei bifosfonati attualmente in commercio è controindicata in caso di CKD di IV-V stadio e sono state riportate solo poche esperienze relativamente all'utilizzo di questa classe di farmaci in corso di nefropatia avanzata. Sono stati descritti diversi casi di alterazione della funzione renale in pazienti sottoposti a terapia con bifosfonati per diverse indicazioni, sia ev che os. Fino al 2011 sono stati riportati almeno sedici casi di disfunzione renale acuta con esito fatale dopo la somministrazione di acido zoledronico e segnalazioni di almeno nove casi di disfunzione renale necessitante di terapia sostitutiva dopo l'infusione di Reclast: la prescrivibilità di acido zoledronico è stata pertanto limitata a pazienti con eGFR $>35 \mathrm{~mL} / \mathrm{min} / 1.73 \mathrm{~m}^{2} .{ }^{16}$ L'acido zoledronico è stato associato sia a necrosi tubulare acuta che a glomerulosclerosi focale e segmentaria (GSFS) in pazienti con mieloma multiplo, malattia di Paget o metastasi ossee. ${ }^{17,18}$ In letteratura sono stati riportati tre casi di insufficienza renale acuta secondaria a somministrazione ev di etidronato. ${ }^{19}$ Più recentemente sono stati riportati sette casi di sindrome nefrosica e insufficienza renale acuta in concomitanza con l'utilizzo di pamidronato in pazienti affetti da mieloma multiplo: in tutti questi casi la diagnosi istologica è stata GSFS collassante, e la sospensione del farmaco è stata accompagnata da una stabilizzazione o da un miglioramento della funzione renale. ${ }^{20}$

Non è ben chiara la patogenesi delle complicanze nefrologiche in corso di terapia con bifosfonati, e sembra che la tossicità renale possa essere differente per le due classi. Relativamente agli amminobifosfonati (pamidronato e alendronato) è probabile che la nefrotossicità possa essere secondaria all'inibizione della via del mavelonato e della prenilazione delle proteine intracellulari. Una quota rilevante di proteine prenilate in condizioni fisiologiche è rappresentata dalla guanosina trifosfatasi, molecola di segnale con implicazioni sulla morfologia mediata dall'organizzazione del ci-toscheletro, la mobilitazione degli endosomi, l'interazione proteina-proteina: è stato dimostrato in vitro che la somministrazione di bifosfonati di questa classe è in grado di indurre apoptosi in cellule umane in coltura ed è stato riportato come il pamidronato sia maggiormente citotossico rispetto ad alendronato e risedronato. ${ }^{21,22} \mathrm{Si}$ ritiene che i bisfosfonati somministrati ev siano più frequentemente associati a nefrotossicità rispetto alle somministrazioni per os, e che tale evento avverso possa essere correlato sia alla dose che alla velocità di infusione. La grave nefrotossicità può essere evitata monitorando la creatininemia prima di ogni trattamento, sospendendo temporaneamente la terapia nel sospetto di un danno renale acuto, e regolando le dosi nei pazienti con già nota malattia renale cronica. ${ }^{23}$

\section{Bifosfonati nella CKD}

La CKD è associata già dagli stadi più precoci ad alterazioni funzionali del metabolismo minerale osseo con il coinvolgimento di numerosi mediatori umorali e cellulari fino a quadri complessi di modifiche strutturali e di alterazione del turnover del tessuto osseo. Le fratture da fragilità risultano essere significativamente superiori nella popolazione uremica rispetto alla popolazione osteoporotica generale l'incremento di tale rischio è verosimilmente legato a diverse condizioni fisiopatologiche contemporaneamente presenti in corso di uremia, tra cui iperparatiroidismo secondario, deficit di vitamina $\mathrm{D}$, amiloidosi, disturbi nutrizionali ed incremento dello stress ossidativo. ${ }^{1,24} \mathrm{Il}$ trattamento dell'osteoporosi nel paziente con nefropatia dal I al III stadio è sostanzialmente sovrapponibile a quanto previsto per la popolazione generale e prevede modifiche dello stile di vita, supplementazione di vitamina D ed eventuale somministrazione di bifosfonati, con relativa bassa probabilità di malattia adinamica dell'osso. Il trattamento diventa più complesso e controverso nel paziente con disfunzione renale severa e terminale. In questa popolazione, infatti, è molto elevato il rischio di sviluppo di malattia adinamica dell'osso in virtù del meccanismo d'azione dei bifo-sfonati e per il rischio di ulteriore compromissione della funzione renale residua. Recenti evidenze suggeriscono peraltro che la malattia adinamica dell'osso, che in precedenza si riteneva tipica dei pazienti uremici, in realtà non è rara già in stadi precoci di malattia renale cronica come conseguenza di un'iniziale resistenza al PTH e per l'azione di altre tossine uremiche. ${ }^{25}$ Alterazioni del metabolismo osseo correlate con basso turnover pertanto non possono essere escluse in nessuno stadio della malattia renale cronica. Nel contesto di una malattia adinamica non si ravvede alcun vantaggio nel procedere alla somministrazione di farmaci che ulteriormente riducono il turnover osseo. La biopsia ossea potrebbe essere utile per guidare il trattamento, ma non è disponibile in tutti i centri e non è ben tollerata dal paziente: indispensabile in questi casi tenere conto dei comuni parametri di laboratorio di metabolismo osseo. ${ }^{26}$ I dati clinici da trial controllati nella popolazione con nefropatia avanzata sono peraltro molto limitati, privando il clinico (ed il paziente) di una risorsa terapeutica di primaria importanza in questo contesto (tabella 2). È ancora incerto il rischio relativo nella popolazione in dialisi di altre complicanze secondarie alla somministrazione di bifosfonati, come l'esofagite, la fibrillazione atriale, le fratture atipiche, l'ipocalcemia e l'infiammazione oculare. ${ }^{27}$

Un caso particolare di utilizzo dei bifosfonati è rappresentato dal trattamento dell'osteoporosi nel paziente sottoposto a trapianto renale. In questi pazienti, la BMD si riduce rapidamente dopo il trapianto; la progressione della patologia ossea è correlata con la 
Tabella 2. Bifosfonati posologia e dose/aggiustamento nella MRC.

\begin{tabular}{|c|c|c|c|c|c|}
\hline Principio attivo & Nome commerciale & Stadio CKD & $\begin{array}{l}\text { Via di } \\
\text { somministrazione }\end{array}$ & $\begin{array}{l}\text { Posologia } \\
\text { (osteoporosi) }\end{array}$ & Posologia in CKD \\
\hline Clodronato & $\begin{array}{l}\text { Clasteon } 400 \mathrm{mg} \mathrm{cp}, 100 \mathrm{mg} \\
3.3 \mathrm{~mL} \mathrm{fl}, 300 \mathrm{mg} / 10 \mathrm{~mL} \mathrm{fl} \\
\text { Clodron } 400 \mathrm{mg} \mathrm{cp}, 100 \\
\mathrm{mg} / 3.3 \mathrm{~mL} \mathrm{fl} \\
\text { Clody } 100 \mathrm{mg} \mathrm{fl}, 300 \mathrm{mg} \mathrm{fl} \\
\text { Difosfonal } 400 \mathrm{mg} \mathrm{cp}, 300 \mathrm{mg} \mathrm{fl} \\
\text { Moticlod } 100 \mathrm{mg} / 3.3 \mathrm{~mL} \mathrm{fl}, 300 \\
\mathrm{mg} / 10 \mathrm{~mL} \\
\text { Ossiten } 400 \mathrm{mg} \mathrm{cp}, 300 \mathrm{mg} \mathrm{fl}\end{array}$ & I, II, III & Os, ev, im & $\begin{array}{l}100 \mathrm{mg} \mathrm{im} \mathrm{I} \mathrm{fl/l4} \\
\text { giorni o } 200 \mathrm{mg} \mathrm{ev} \mathrm{I} \\
\text { fl/mese }\end{array}$ & $\begin{array}{l}\text { Riduzione } 50 \% \text { con } \\
\mathrm{ClCr} \text { tra } 60 \text { e } 30 \\
\mathrm{~mL} / \mathrm{min}\end{array}$ \\
\hline Alendronato & $\begin{array}{l}\text { Alendros, Adronat, Fosamax, } \\
\text { Dronal, Genalen } 10 \mathrm{mg} \mathrm{cp} \mathrm{o} \\
70 \mathrm{mg} \mathrm{cp}\end{array}$ & I, II, III & Os & $70 \mathrm{mg} I \mathrm{cp} / \mathrm{sett}$ & $\begin{array}{l}\text { Nessuna riduzione } \\
\text { in } \mathrm{CKD} \text { se } \mathrm{ClCr} \\
<35 \mathrm{~mL} / \mathrm{min}\end{array}$ \\
\hline Risedronato & $\begin{array}{l}\text { Actonel } 5 \mathrm{mg}, 35 \mathrm{mg} \circ 75 \mathrm{mg} \\
\text { cp } \\
\text { Optinate } 5 \mathrm{mg}, 35 \mathrm{mg} \circ 75 \\
\mathrm{mg} \mathrm{cp}\end{array}$ & I, II, III & Os & $35 \mathrm{mg}$ I cp/sett & $\begin{array}{l}\text { Nessuna riduzione } \\
\text { in } \mathrm{CKD} \text { se } \mathrm{ClCr} \\
<30 \mathrm{~mL} / \mathrm{min}\end{array}$ \\
\hline Ibadronato & $\begin{array}{l}\text { Bondenza } 2.5 \mathrm{mg} \mathrm{cP} \\
\text { Bondronat } 6 \mathrm{mg} / 6 \mathrm{~mL} \mathrm{fl}\end{array}$ & I, II, III & Os, ev & $\begin{array}{l}150 \mathrm{mg} \mathrm{I} \mathrm{cp} / \mathrm{mese} \text { o } \\
6 \mathrm{mg} \mathrm{ev} \mathrm{I} \mathrm{fl/mese}\end{array}$ & $\begin{array}{l}\text { Nessuna riduzione } \\
\text { in } \mathrm{CKD} \text { se } \mathrm{ClCr} \\
<30 \mathrm{~mL} / \mathrm{min}\end{array}$ \\
\hline Pamidronato $(*)$ & $\begin{array}{l}\text { Amidrox } 3 \mathrm{mg} / \mathrm{mL} \text {, fl da } 5 \mathrm{~mL} \text {, } \\
10 \mathrm{~mL}, 20 \mathrm{~mL}, 30 \mathrm{~mL} \\
\text { Aredia } 15 \mathrm{mg} / 5 \mathrm{~mL}, 30 \mathrm{mg} / 10 \\
\mathrm{~mL}, 60 \mathrm{mg} / 10 \mathrm{~mL}, 90 \mathrm{mg} / 10 \\
\mathrm{~mL} \\
\text { Texapami } 3 \mathrm{mg} / \mathrm{mL}, 6 \mathrm{mg} / \mathrm{mL} \text { e } \\
9 \mathrm{mg} / \mathrm{mL}\end{array}$ & I, II, III & $\mathrm{Ev}$ & $\begin{array}{l}\text { Da } 15 \text { a } 90 \mathrm{mg} \text { ev in } \\
\text { due ore, ogni quattro } \\
\text { settimane }\end{array}$ & $\begin{array}{l}\text { Nessuna riduzione } \\
\text { in } \mathrm{CKD} \text { se } \mathrm{ClCr} \\
<30 \mathrm{~mL} / \mathrm{min}\end{array}$ \\
\hline $\begin{array}{l}\text { Acido } \\
\text { zoledronico }\end{array}$ & Zometa 4 mg/l 00 mL fl & I, II, III & Ev & $5 \mathrm{mg}$ ev I fl/anno & $\begin{array}{l}\text { Nessuna riduzione } \\
\text { in } \mathrm{CKD} \text { se } \mathrm{ClCr} \\
<35 \mathrm{~mL} / \mathrm{min}\end{array}$ \\
\hline
\end{tabular}

(*):non approvato in Italia per il trattamento dell'osteoporosi; la posologia si riferisce al trattamento dell'ipercalcemia neoplastica da metastasi ossee e mieloma multiplo.

cp: compresse; fl: fiale; per os: orale; ev: endovena; im: intramuscolo.

durata della malattia di base, la durata della dialisi, il regime immunosoppressivo e la somministrazione di steroidi. È stata descritta una perdita di BMD valutata a livellolombare fino al $6.8 \%$ in un anno, significativamente superiore all' $1.7 \%$ della popolazione in menopausa. Le Linee Guida KDIGO suggeriscono la somministrazione di bifosfonati ma il livello di evidenza attribuito a tale affermazione è basso. ${ }^{28}$ Recenti revisioni degli studi presenti in letteratura hanno permesso di evidenziare che la terapia con bifosfonati nel paziente trapiantato non è associata ad incremento dell'incidenza di disfunzione del trapianto, mentre è stato dimostrato un miglioramento significativo della BMD sia a livello femorale che lombare; a tale miglioramento non è seguita una riduzione dell'incidenza di nuove fratture. ${ }^{29}$ L'utilizzo di bifosfonati nei pazienti trapiantati non è tuttavia del tutto privo di problematiche. Recentemente, uno studio condotto su un limitato numero di pazienti trapiantati ha permesso di evidenziare che, ad un anno dal trapianto, esiste una tendenza alla malattia adinamica dell'osso come testimoniato sia dai parametri biochimici che dalle evidenze bioptiche contestuali. ${ }^{30}$ Queste nuove evidenze suggeriscono prudenza nella prescrizione di bifosfonati in tale popolazione, per la quale è necessario il costante monitoraggio dei parametri biochimici di turnover osseo e l'eventuale sospensione della terapia in caso di evidenza di basso turnover. 
La calcifilassi e le calcificazioni vascolari rappresentano complicanze tardive della $\mathrm{CKD}$, associate alle note alterazioni del metabolismo osseo secondarie all'iperparatiroidismo. È stato riscontrato che i bifosfonati inibiscono le calcificazioni vascolari ed è stato riportato che etidronato e pamidronato risultano essere utili nel trattamento della calcifilassi. I meccanismi farmacologici attraverso i quali si verificano questi potenziali effetti favorevoli rimangono non definiti. È stato ipotizzato che l'inibizione del riassorbimento osseo riduca la mobilizzazione di calcio dal tessuto osseo e ne limiti la deposizione vascolare, con particolare riferimento ai modelli di CKD-MBD ad elevato turnover osseo. ${ }^{31}$ Queste osservazioni, condotte sia nell'animale da esperimento che sull'uomo, sono molto suggestive, ma al momento rimangono puramente speculative poiché non sono supportate da specifici trial clinici.

\section{Alendronato}

L'alendronato è considerato uno dei farmaci di prima scelta per il trattamento dell'osteoporosi. Jamal e colleghi ${ }^{32}$ hanno pubblicato un'analisi post-hoc sull'outcome renale rielaborando i dati dello studio FIT, un trial alendronato vs. placebo su oltre 6,000 donne con osteoporosi postmenopausa. Gli Autori hanno dimostrato che non si sono verificate variazioni significative della funzione renale nei 36 mesi di follow-up e non sono stati segnalati eventi avversi nanche nei pazienti con CKD al IV e V stadio non in dialisi. Yanik e colleghi ${ }^{33}$ hanno invece pubblicato uno studio retrospettivo in cui hanno confrontato pazienti sottoposti a terapia con alendronato, risedronato $\mathrm{o}$ raloxifene: la media dei valori di creatinina ed eGFR non variavano significativamente rispetto al basale.

\section{Risedronato}

Miller e colleghi ${ }^{34}$ hanno condotto un'analisi post-hoc su nove trial clinici relativi all'uso del risedronato per os per il trattamento dell'osteoporosi in cui hanno dimostrato che, rispetto al placebo, non si osservavano variazioni statisticamente significative della funzione renale ri-spetto al basale. Inoltre, in pazienti con CKD moderata o severa (III vs. IV stadio), non si riscontravano nei pazienti trattati con risendronato rispetto a quelli trattati con placebo differenze significative per eventi avversi. Gli stessi autori, valutando i risultati di precedenti studi, hanno concluso in un'altra review che l'utilizzo di risedronato o aledronato non causa alterazioni di rilievo nella funzione renale. ${ }^{35}$

\section{Ibadronato}

L'ibadronato, somministrato per os, non sembra essere associato ad alcuna modifica clinicamente rilevante dei parametri di funzione renale rispetto al basale, come dimostrato da Chesnut e colleghi in uno studio in doppio cieco vs. placebo con un follow-up esteso a tre anni dall'inizio del trattamento. ${ }^{36}$

Delmas e colleghi hanno pubblicato i risultati di uno studio randomizzato in doppio cieco con follow-up di 2 mesi su quasi 1,400 pazienti affetti da osteoporosi postmenopausa divisi in tre gruppi di trattamento: trattati con ibadronato ev con due differenti posologie o ibadronato per os. Il $50 \%$ di questi pazienti erano affetti da CKD definita da $\mathrm{CrCl}<60 \mathrm{~mL} / \mathrm{min}$. Al termine del follow-up previsto, la percentuale dei pazienti con declino significativo della funzione renale era sovrapponibile nei tre gruppi (media 15.16\%). ${ }^{37}$ In assenza di casi di IRA, le variazioni di creatinina non erano correlate con la somministrazione del farmaco. Sanchez e colleghi hanno reclutato e valutato prospetticamente 69 pazienti con trapianto di rene da oltre un anno, con funzione renale stabile, e sottoposti a trattamento con ibandronato $150 \mathrm{mg} /$ mese per os ( $\mathrm{n}=35)$ o risedronato $35 \mathrm{mg} / \mathrm{sett}$ per os $(\mathrm{n}=34)$. Al termine del follow-up non vi era nessuna modifica significativa dei valori di creatinina nei due gruppi, né nei parametri di metabolismo minerale osseo né di BMD, con sostanziale equivalenza dei due schemi di trattamento in termini di efficacia e sicurezza. ${ }^{38}$

\section{Pamidronato}

È stato evidenziato un lieve incremento di sCreat dopo infusione, ma non sono state osservate variazioni significative di creatinina al termine del follow-up. ${ }^{39}$

\section{Acido zoledronico}

L'efficacia e la sicurezza renale dell'acido zoledronico in somministrazione ev ( $5 \mathrm{mg}$ in $15 \mathrm{~min})$ è stata confrontata con il placebo nello studio HORIZON-PTF, i cui risultati sono stati pubblicati nel 2008 da Boonen e colleghi ${ }^{40}$ su un vasto campione di donne con osteoporosi post-menopausa. In questo studio prospettico randomizzato in doppio cieco sono stati esclusi i pazienti affetti da proteinuria o da CKD di stadio IV-V. La notevole estensione del follow-up (tre anni) ha permesso di dimostrare come la somministrazione di questo farmaco non sia associata a modifiche significative della funzione renale o ad un incremento degli eventi avversi renali rispetto al placebo, mentre la riduzione dell'eGFR è stata correlata con l'aumentare dell'età. ${ }^{35}$ Risultati simili erano già stati anticipati dal trial HORIZON-RFT, eseguito su 2,017 pazienti con storia di fratture patologiche e sottoposti a trattamento con acido zoledronico ev o placebo, senza differenze significative di eventi avversi di interesse renale tra $\mathrm{i}$ due gruppi. ${ }^{41}$ Nello studio HORIZON-GIO, che ha reclutato 833 pazienti affetti da osteoporosi secondaria 
a glucocorticoidi, è stato valutato l'outcome a 12 mesi dopo singola somministrazione di acido zoledronico 5 $\mathrm{mg}$ ev confrontato con risedronato $5 \mathrm{mg} /$ die per os. In questo studio si sono verificati due casi di IRA nel gruppo trattato con risedronato ed un caso nel gruppo trattato con acido zoledronico, mentre una riduzione significativa della funzione renale, definita da incrementi di creatininemia $>0.5 \mathrm{mg} / \mathrm{dL}$ rispetto al basale, è stata osservata in quattro pazienti nei due gruppi. Gli Autori hanno attribuito la disfunzione renale osservata alle comorbilità dei pazienti. ${ }^{42}$ Bounameaux e colleghi hanno pubblicato i dati su 1,199 uomini con osteoporosi trattati con acido zoledronico o placebo: gli eventi avversi renali si dimostrarono essere più frequenti nel gruppo di pazienti trattati con placebo. ${ }^{43}$

\section{Discussione}

Le indicazioni all'utilizzo dei bifosfonati nell'adulto sono rappresentate dal trattamento e dalla prevenzione dell'osteoporosi, dall'ipercalcemia correlata con neoplasie metastatiche, dal mieloma multiplo, dalla malattia di Paget dell'osso ed inoltre, relativamente all'età pediatrica, dall'osteogenesi imperfetta. L'escrezione renale, comune a tutte le molecole di questa classe di farmaci, condiziona l'esecuzione di trial clinici comprendenti pazienti affetti da severa riduzione della funzione renale. Per tale motivo, la somministrazione di alendronato, risedronato e ibandronato è sconsigliata in pazienti affetti da CKD di IV-V stadio in terapia conservativa, mentre la somministrazione di acido zoledronico ev è controindicata per $\mathrm{CrCl}<35 \mathrm{~mL} / \mathrm{min} .{ }^{11}$ La mancanza di evidenze cliniche sull'utilizzo di questi farmaci in corso di CKD avanzata ne limita fortemente l'utilizzo in questi pazienti. In letteratura sono stati riportati diversi casi di IRA e sindrome nefrosica correlati con la somministrazione ev di bifosfonati, in particolare acido zoledronico. ${ }^{43}$ Studi più recenti hanno permesso di chiarire che il danno renale conseguente alla somministrazione di questi farmaci risulta essere correlato sia con la dose che con la velocità di infusione: ${ }^{44}$ gli Autori hanno dimostrato che la somministrazione di uguale dosaggio di acido zoledronico ev con velocità di infusione ridotta di un terzo permette di ridurre significativamente il rischio relativo di IRA e di ottenere un profilo di sicurezza renale pari alla somministrazione di aledronato per os in coorti di pazienti sottoposti a trattamento per mieloma multiplo, carcinoma mammario e carcinoma prostatico metastatico. I risultati dei trial hanno evidenziato come la disfunzione renale possa essere transitoria, mentre le biopsie renali hanno evidenziato come anche nei casi più impegnativi, IRA e sindrome nefrosica, la sospensione della terapia porta ad una stabilizzazione o ad un miglioramento del quadro clinico. ${ }^{45,46}$ Sebbene ad oggi non siano stati riportati trial clinici relativi all'uso dei bifosfonati su pazienti con CKD grave, nei trials disponibili è stato riportato come l'utilizzo di alendronato, risedronato e ibadronato per os non sia associato a modifiche significative della funzione renale rispetto ai gruppi trattati con placebo. ${ }^{33,34}$ Inoltre uno studio prospettico comprendente oltre 120,000 soggetti con più di 65 anni, di cui il $40 \%$ affetto da CKD, trattati con bifosfonati per os per frattura patologica ha evidenziato come non esista nessun aumento di rischio relativo di danno renale acuto durante il follow-up esteso a 90 giorni. ${ }^{48}$ Inoltre, non vi era nessuna differenza statisticamente significativa nell'outcome dei pazienti del sottogruppo con CKD già nota. ${ }^{47}$ La valutazione delle evidenze dei trial clinici e delle review pubblicate negli ultimi anni (tabella 3) permette di considerare i bifosfonati farmaci relativamente sicuri, con favorevole rapporto rischio/beneficio anche nel paziente con insufficienza renale moderata in virtù della riduzione significativa del rischio di fratture e della perdita di massa ossea a fronte di un incerto rischio di aggravamento della nefropatia, purché usati con posologia appropriata ed in pazienti stabili; non sono disponibili valutazioni puntuali sul rischio legato alla condizione di "frozen bone". L'estensione dei trial a pazienti con insufficienza renale cronica moderata ha permesso di valutare l'insussistenza di controindicazioni specifiche per questa classe di farmaci. Non esistono al momento dati riguardanti la prescrivibilità di bisfosfonati nell'insufficienza renale terminale. Ad integrazione delle classiche terapie per l'osteoporosi e per la CKD-MBD, non è attualmente ancora chiaro nella popolazione nefropatica il profilo di sicurezza di alcuni nuovi farmaci biologici quali il denosumab, il teriparatide e il più recente romosuzumab. Il teriparatide trova indicazione nell'osteoporosi severa non trattabile o non responsiva ad altre terapie; tra gli effetti collaterali è stato segnalato iperuricemia, ipercalcemia e ipercalciuria ed è controindicato nel paziente con storia di nefrolitiasi; l'effetto sull'osso è di tipo anabolico. Il denosumab è indicato per il trattamento dell'osteoporosi non trattabile o non responsiva ad altre terapie, nei pazienti ad alto rischio di fratture e nell'ipercalcemia neoplastica non responsiva ai bifosfonati, l'ipocalcemia ne rappresenta l'effetto collaterale più frequente; è stato inoltre descritto un effetto di tipo anti-riassorbitivo nel tessuto osseo. Il teriparatide è un farmaco ancora in fase sperimentale, con azione anti-riassorbitiva e anabolica, per il quale non si dispone in atto di sufficienti dati sul profilo di sicurezza. In prospettiva, è stato descritto che questi farmaci potrebbero essere usati in maniera mirata per bilanciare il metabolismo osseo nelle diverse manifestazioni di osteodistrofia renale. ${ }^{48}$

\section{Conclusioni}

I bifosfonati rappresentano un'importante classe di farmaci nella gestione del paziente con osteoporosi ed altre frequenti malattie ossee, spesso coesistenti con 
Tabella 3. Bifosfonati ed insufficienza renale, studi clinici.

\begin{tabular}{|c|c|c|c|c|c|c|c|c|}
\hline Autore & Anno & $\mathrm{n}$ & Farmaco & Dose farmaco & Disegno & Outcome renale & $\begin{array}{l}\text { Outcome metabolismo } \\
\text { osseo }\end{array}$ & $\begin{array}{l}\text { Follow-up } \\
\text { (mesi) }\end{array}$ \\
\hline $\begin{array}{l}\text { Chesnut } \\
(33)\end{array}$ & 2004 & 2946 & $\begin{array}{l}\text { lbandronato vs. } \\
\text { placebo }\end{array}$ & $\begin{array}{l}2.5 \mathrm{mg} / \mathrm{die} \\
\text { per os } \times 12 \mathrm{gg} \\
\text { ogni } 3 \text { mesi }\end{array}$ & $\begin{array}{l}\text { Trial doppio } \\
\text { cieco }\end{array}$ & $\begin{array}{l}\text { Assenti variazioni } \\
\text { significative della } \\
\text { creatinina }\end{array}$ & $\begin{array}{l}\text { Riduzione incidenza } \\
\text { totale di nuove fratture } \\
\text { nei gruppi trattati vs } \\
\text { placebo, simile incidenza } \\
\text { di fratture non } \\
\text { vertebrali }\end{array}$ & 36 \\
\hline Miller (32) & 2005 & 9883 & $\begin{array}{l}\text { Risedronato vs. } \\
\text { placebo }\end{array}$ & $\begin{array}{l}5 \mathrm{mg} / \text { die } \times 3 \\
\text { anni }\end{array}$ & $\begin{array}{l}\text { Analisi post- } \\
\text { hoc }\end{array}$ & $\begin{array}{l}\text { Assenti variazioni } \\
\text { significative della } \\
\text { creatinina }\end{array}$ & $\begin{array}{l}\text { Riduzione dell'incidenza } \\
\text { di fratture vertebrali e } \\
\text { mantenimento di BMD }\end{array}$ & 24 \\
\hline $\begin{array}{l}\text { Delmas } \\
\text { (34) }\end{array}$ & 2006 & 1395 & $\begin{array}{l}\text { lbandronato vs. } \\
\text { lbandronato vs. } \\
\text { lbadronato }\end{array}$ & $\begin{array}{l}2 \mathrm{mg} \text { ev. ogni } \\
\text { due mesi vs. } \\
3 \mathrm{mg} \text { ev. ogni } \\
3 \text { mesi vs. } 2.5 \\
\text { mg/die per os }\end{array}$ & $\begin{array}{l}\text { Trial doppio } \\
\text { cieco }\end{array}$ & $\begin{array}{l}\text { No IRA, I } 2 \text { casi di } \\
\text { peggioramento di } \\
\text { CKD }\end{array}$ & $\begin{array}{l}\text { Somministrazione } \\
\text { ev superiore vs a } \\
\text { somministrazione per } \\
\text { os in aumento di BMD a } \\
\text { livello lombare }\end{array}$ & 24 \\
\hline Lyles (39) & 2007 & 2127 & $\begin{array}{l}\text { Acido } \\
\text { zoledronico vs. } \\
\text { placebo }\end{array}$ & $\begin{array}{l}5 \mathrm{mg} \mathrm{ev} \\
\text { annuali }\end{array}$ & $\begin{array}{l}\text { Trial doppio } \\
\text { cieco }\end{array}$ & $\begin{array}{l}\text { Assenti modifiche } \\
\text { significative di GFR }\end{array}$ & $\begin{array}{l}\text { Riduzione del rischio } \\
\text { di fratture del } 35 \% \\
\text { nei pazienti trattati vs } \\
\text { placebo, riduzione della } \\
\text { mortalità per tutte } \\
\text { le cause nei pazienti } \\
\text { trattati }\end{array}$ & 36 \\
\hline Jamal (30) & 2007 & 6438 & $\begin{array}{l}\text { Alendronato vs. } \\
\text { placebo }\end{array}$ & $\begin{array}{l}5 \mathrm{mg} / \text { die } \times 2 \\
\text { anni poi } 10 \\
\mathrm{mg} / \mathrm{die}\end{array}$ & $\begin{array}{l}\text { Analisi post } \\
\text { hoc }\end{array}$ & $\begin{array}{l}\text { Incremento non } \\
\text { significativo della } \\
\text { creatinina }\end{array}$ & $\begin{array}{l}\text { Miglioramento di BMD } \\
\text { e riduzione di nuove } \\
\text { fratture ossee anche nei } \\
\text { pazienti con funzione } \\
\text { renale ridotta }\end{array}$ & 36 \\
\hline Yanik (3I) & 2007 & 127 & $\begin{array}{l}\text { Alendronato vs. } \\
\text { risedronato }\end{array}$ & $\begin{array}{l}70 \mathrm{mg} / \mathrm{sett} \mathrm{vs} \\
35 \mathrm{mg} / \mathrm{set}\end{array}$ & $\begin{array}{l}\text { Analisi post } \\
\text { hoc }\end{array}$ & $\begin{array}{l}\text { Assenti modifiche } \\
\text { significative di GFR }\end{array}$ & Dati non disponibili & 12 \\
\hline $\begin{array}{l}\text { Boonen } \\
(37)\end{array}$ & 2008 & 7765 & $\begin{array}{l}\text { Acido } \\
\text { zoledronico vs. } \\
\text { placebo }\end{array}$ & $\begin{array}{l}5 \mathrm{mg} \mathrm{ev} \\
\text { annuali }\end{array}$ & $\begin{array}{l}\text { Trial doppio } \\
\text { cieco }\end{array}$ & $\begin{array}{l}\text { Assenti modifiche } \\
\text { significative di } \\
\text { creatinina o GFR }\end{array}$ & Dati non disponibili & 36 \\
\hline Reid (40) & 2009 & 833 & $\begin{array}{l}\text { Acido } \\
\text { zoledronico vs. } \\
\text { risedronato }\end{array}$ & $\begin{array}{l}5 \mathrm{mg} \text { ev } \\
\text { annuali vs. } 5 \\
\mathrm{mg} / \text { die }\end{array}$ & $\begin{array}{l}\text { Trial doppio } \\
\text { cieco }\end{array}$ & $\begin{array}{l}3 \text { IRA per causa } \\
\text { diversa dal farmaco }\end{array}$ & $\begin{array}{l}\text { Acido zoledronico } \\
\text { non inferiore e } \\
\text { probabilmente } \\
\text { superiore vs } \\
\text { risedronato nel } \\
\text { migliorare BMD a livello } \\
\text { lombare nei sottogruppi } \\
\text { analizzati }\end{array}$ & 12 \\
\hline Black (38) & 2012 & 1199 & $\begin{array}{l}\text { Acido } \\
\text { zoledronico vs } \\
\text { placebo }\end{array}$ & $\begin{array}{l}5 \mathrm{mg} \text { ev } \\
\text { annuali }\end{array}$ & Prospettico & $\begin{array}{l}\text { Assenti modifiche } \\
\text { significative di } \\
\text { creatinina o GFR }\end{array}$ & Dati non disponibili & 72 \\
\hline $\begin{array}{l}\text { Palomo } \\
\text { (15) }\end{array}$ & 2015 & 14 & Pamidronate ev & $\begin{array}{l}\mathrm{I} \mathrm{mg} / \mathrm{kg} \times 3 \mathrm{gg} \\
\text { ogni } 4 \text { mesi vs } \\
2 \mathrm{mg} / \mathrm{kg} \text { ogni } 4 \\
\text { mesi }\end{array}$ & Prospettico & $\begin{array}{l}\text { Lieve incremento } \\
\text { di creatinina dopo } \\
\text { infusione }\end{array}$ & $\begin{array}{l}\text { Non significative } \\
\text { differenze di Z-score } \\
\text { lombare nei due gruppi }\end{array}$ & 12 \\
\hline $\begin{array}{l}\text { Sanchez } \\
\text { Escuredo } \\
\text { (35) }\end{array}$ & 2015 & 69 & $\begin{array}{l}\text { I=lbadronato } \\
\text { vs. risedronato }\end{array}$ & $\begin{array}{l}150 \text { mg vs } 35 \\
\text { mg }\end{array}$ & Prospettico & $\begin{array}{l}\text { Assenti modifiche } \\
\text { significative di } \\
\text { creatinina }\end{array}$ & $\begin{array}{l}\text { Nessuna differenza } \\
\text { significativa per BMD } \\
\text { nei due gruppi di } \\
\text { trattamento }\end{array}$ & 12 \\
\hline
\end{tabular}

quadri di nefropatia cronica. Gli studi clinici hanno dimostrato un ottimo profilo di sicurezza renale (eventi acuti, progressione nefropatia) limitatamente ai pazienti stabili con valori di eGFR $>30-35 \mathrm{~mL} / \mathrm{min} / 1.73 \mathrm{~m}^{2} \mathrm{ma}$ non sono stati riportati trial controllati per funzioni più compromesse, tenuto conto anche del meccanismo di escrezione, motivo per cui sono generalmente controindicati nei pazienti nefropatici avanzati e 
terminali. Al fine di stabilire il reale rapporto rischio/ beneficio di questi farmaci per eGFR $<30 \mathrm{~mL} / \mathrm{min} / 1.73$ $\mathrm{m}^{2}$, sarà necessario procedere a trial clinici controllati in questa specifica popolazione, per eventuale ampliamento della prescrivibilità con potenziali benefici in termini di miglioramento di morbilità.

\section{Dichiarazione di conflitto di interessi}

Gli Autori dichiarano di non avere conflitto di interessi.

\section{Contributi economici ali Autori}

Gli Autori dichiarano di non avere ricevuto sponsorizzazioni economiche per la preparazione dell'articolo e che esso non contiene immagini e/o tabelle soggette ad autorizzazioni.

\section{Bibliografia}

1. Fusaro M, Aghi A, Mereu MC and Giusti A. Fratture da fragilità nella Malattia Renale Cronica. G Ital Nefrol 2017; 34 (Nov-Dec). pii: 2017-vol 6.

2. Jha V, Garcia-Garcia $G$ and Iseki K. Chronic kidney disease: global dimension and perspectives. Lancet 2013; 382(9888):260-272.

3. WHO Scientific Group on the Prevention and Management of Prevention and management of osteoporosis. World Health Organ Tech Rep Ser 2003; 921:1-164.

4. NIH Consensus Development Panel on Osteoporosis Prevention, Diagnosis, and Therapy, Osteoporosis prevention, diagnosis, and therapy. JAMA 2001; 285(6): 785-795.

5. Felsenberg D and Boonen S. The bone quality framework: determinants of bone strength and their interrelationships, and implications for osteoporosis management. Clin Ther 2005; 27(1):1-11.

6. Isakova $\mathrm{T}$, Nickolas $\mathrm{TL}$, Denburg $\mathrm{M}$, et al. KDOQI US commentary on the 2017 KDIGO clinical practice guideline update for the diagnosis, evaluation, prevention, and treatment of chronic kidney disease-mineral and bone disorder (CKD-MBD). Am J Kidney Dis 2017; 70(6): 737-751.

7. Ott SM. Bone strength: more than just bone density. Kidney Int 2016; 89(1):16-94.

8. Ott SM, Pharmacology of Bisphosphonates in Patients with Chronic Kidney Disease. Sem Dial 2015; 28(4):363-369.

9. Corrado A and Cantatore FP. I bifosfonati: caratteristiche chimiche, effetti biologici scheletrici ed effetti extrascheletrici. Reumat 2005; 57(3):142-153.

10. Choudhury D and Ahmed Z. Drug-associated renal dysfunction and injury. Nat Clin Pract Nephrol 2006; 2:80-91.

11. Miller PD, Jamal SA, Evenepoel P, Eastell R and Boonen S. Renal safety in patients treated with bisphosphonates for osteoporosis: a review. J Bone Mine Res 2013; 28(10): 2049-2059.

12. Lin JH, Chen IW, Deluna FA and Hichens M. Renal handling of alendronate in rats. An uncharacterized renal transport system. Drug Metab Dispos 1992; 20(4): 608-613.
13. Brandi ML. Use of clodronic acid in mineral metabolism conditions: state of the art in 2000.Minerva Med 2001;92(4): 251-268.

14. Chesnut CH III, Skag A, Christiansen C, et al. Effects of oral ibandronate administered daily or intermittently on fracture risk in postmenopausal osteoporosis. J Bone Miner Res 2004; 19:1241-1249.

15. Palomo T, Andrade MC, Peters BS, et al. Evaluation of a modified pamidronate protocol for the treatment of osteogenesis imperfecta. Calcif Tissue Int 2015; 98(1): 42-48.

16. U.S. Food and Drug Administration (FDA). FDA Drug Safety Communication: New contraindication and updated warning on kidney impairment for Reclast (zoledronic acid). Silver Spring (MD): U.S. Food and Drug Administration; 2011 https://www.fda.gov/Drugs/DrugSafety/ucm270199. htm (accessed 10 January 2019).

17. Bodmer M, Amico P, Mihatsch MJ, et al. Focal segmental glomerulosclerosis associated with long-term treatment with zoledronate in a myeloma patient. Nephrol Dial Transplant 2007; 22:2366-2370.

18. Joensuu TK. Renal toxicity following zoledronic acid reversed with ibandronate in a prostate cancer patient with bone metastases. Urol Int 2008; 80:448-450.

19. Bounameaux HM, Schifferli J, Montani JP, et al. Renal failure associated with intravenous diphosphonates. Lancet $1983 ; 1: 471$.

20. Markowitz GS, Appel GB, Fine PL, et al. Collapsing focal segmental glomerulosclerosis following treatment with high-dose pamidronate. J Am Soc Nephrol 2001; 12(6):1164-1172.

21. Suri S, Monkkonen J, Taskinen M, et al. Nitrogencontaining bisphosphonates induce apoptosis of Caco-cells in vitro by inhibiting the mevalonate pathway: a model of bisphosphonate-induced gastrointestinal toxicity. Bone 2001; 29:336-343.

22. Benford HL, Frith JC, Auriola S, et al. Farnesol and geranylgeraniol prevent activation of caspases by aminobisphosphonates: biochemical evidence for two distinct pharmacological classes of bisphosphonate drugs. Mol Pharmacol 1999; 56:131-140.

23. Perazella MA1 and Markowitz GS. Bisphosphonate nephrotoxicity. Kidney Int 2008; 74(11):1385-1393.

24. Nitta K, Yajima A and Tsuchiya K. Management of osteoporosis in chronic kidney disease, Intern Med 2017; 56(24):3271-3276.

25. Drüeke TB and Massy ZA. Changing bone patterns with progression of chronic kidney disease. Kidney Int 2016; 89(2):289-302.

26. Miller PD. The role of bone biopsy in patients with chronic renal failure, Clin J Am Soc Nephrol 2008; 3(Suppl 3): S140-S150.

27. Connelly K, Collister D and Tangri N. Fracture risk and treatment in chronic kidney disease. Curr Opin Nephrol Hypertens 2018; 27(3):221-225.

28. Sadideen H, Covic A and Goldsmith D. Mineral and bone disorder after renal transplantation: a review. Int Urol Nephrol 2008; 40(1):171-184.

29. Toth-Manikowski SM, Francis JM, Gautam A, et al. Outcomes of bisphosphonate therapy in kidney transplant 
recipients: a systematic review and meta-analysis. Clin Transplant 2016; 30(9):1090-1096.

30. Evenepoel P, Behets GJ, Viaene L, et al. Bone histomorphometry in de novo renal transplant recipients indicates a further decline in bone resorption 1 year posttransplantation. Kidney Int 2017; 91(2):469-476.

31. Persy V, De Broe M and Ketteler M. Bisphosphonates prevent experimental vascular calcification: Treat the bone to cure the vessels? Kidney Int 2006; 70(9):1537-1538.

32. Jamal SA, Bauer DC, Ensrud KE, et al. Alendronate treatment in women with normal to severely impaired renal function: an analysis of the fracture intervention trial. $J$ Bone Miner Res 2007; 22:503-508.

33. Yanik B, Bavbek N, Yanik T, et al. The effect of alendronate, risedronate, and raloxifene on renal functions, based on the Cockcroft and Gault method, in postmenopausal women. Ren Fail 2007; 29:471-476.

34. Miller PD, Roux C, 20. Boonen S, et al. Safety and efficacy of risedronate in patients with age-related reduced renal function as estimated by the Cockcroft and Gault method: a pooled analysis of nine clinical trials. $J$ Bone Miner Res 2005; 20:2105-2115.

35. Black DM, Miller PD, Hruska J, et al. Renal effects of treatment with zoledronic acid: results from the HORIZONPFT extension study. Osteoporos Int 2012; 23:S197-198.

36. Chesnut CH III, Skag A, Christiansen C, et al. Effects of oral ibandronate administered daily or intermittently on fracture risk in postmenopausal osteoporosis. J Bone Miner Res 2004; 19:1241-1249.

37. Delmas PD, Adami S, Strugala C, et al. Intravenous ibandronate injections in postmenopausal women with osteoporosis: one-year results from the dosing intravenous administration study. Arthritis Rheum 2006; 54:1838-1846.

38. Sanchez Escuredo A, Fuster D, Rubello D, et al. Monthly ibandronate versus weekly risedronate treatment for low bone mineral density in stable renal transplant patients. $\mathrm{Nucl}$ Med Commun 2015; 36(8):815-884.
39. Palomo T, Andrade MC, Peters BS, et al. Evaluation of a Modified Pamidronate Protocol for the Treatment of Osteogenesis Imperfecta. Calcif Tissue Int 2016; 98(1): 42-48.

40. Boonen S, Sellmeyer DE, Lippuner K, et al. Renal safety of annual zoledronic acid infusions in osteoporotic postmenopausal women. Kidney Int 2008; 74:641-648.

41. Lyles KW, Colon-Emeric CS, Magaziner JS, et al. Zoledronic acid and clinical fractures and mortality after hip fracture. N Engl J Med 2007; 357:1799-1809.

42. Reid DM, Devogelaer JP, Saag K, et al. Zoledronic acid and risedronate in the prevention and treatment of glucocorticoidinduced osteoporosis (HORIZON): a multicentre, double-blind, double-dummy, randomised controlled trial. Lancet 2009; 373:1253-1263.

43. Bounameaux HM, Schifferli J, Montani JP, et al. Renal failure associated with intravenous diphosphonates. Lancet $1983 ; 1: 471$.

44. Perazella MA and Markowitz GS, Bisphosphonate nephrotoxicity. Kidney Int 2008; 74(11):1385-1393.

45. Rosen LS, Gordon D, Kaminski M, et al. Long-term efficacy and safety of zoledronic acid compared with pamidronate disodium in the treatment of skeletal complications in patients with advanced multiple myeloma or breast carcinoma: a randomized, double-blind, multicenter, comparative trial. Cancer 2003; 98:1735-1744.

46. Saad F, Gleason DM, Murray R, et al. Zoledronic Acid Prostate Cancer Study Group. A randomized, placebocontrolled trial of zoledronic acid in patients with hormonerefractory metastatic prostate carcinoma. J Natl Cancer Inst 2002; 94:1458-1468.

47. Shih AW, Weir MA, Clemens KK, et al. Oral bisphosphonate use in the elderly is not associated with acute kidney injury. Kidney Int 2012; 82:903-908.

48. Tartaglione L, Pasquali M, Rotondi S, et al. Positioning novel biologicals in CKD-mineral and bone disorders. $J$ Nephrol 2017; 30(5):689-699. 